UVX 2008 (2009) 71-76

(C) EDP Sciences, 2009

DOI: $10.1051 /$ uvx/2009012

\title{
Absorption X près des seuils (XANES, EXAFS) pour l'étude de la matière dense et tiède
}

\author{
M. Harmand, C. Fourment, S. Hulin, J.J. Santos, \\ O. Peyrusse et F. Dorchies \\ Université de Bordeaux-CNRS-CEA, Centre Lasers Intenses et Applications (CELIA), \\ 33405 Talence, France
}

\begin{abstract}
Résumé. Afin de réaliser des expériences de spectroscopie d'absorption résolue en temps, nous avons développé une source $X$ intense et de large bande spectrale. Pour cela, nous avons utilisé le rayonnement de couche $\mathrm{M}$ de plasmas de $\mathrm{Z}$ élevés, produits par un laser $\mathrm{kHz}$, de durée d'impulsion variable (30 fs$3 \mathrm{ps)}$, aboutissant à une intensité sur cible de $10^{15-17} \mathrm{~W} / \mathrm{cm}^{2}$. Les spectres d'émission expérimentaux ont été étudiés dans la gamme d'énergie $1,50-1,75 \mathrm{keV}$ et optimisés pour sonder le flanc $\mathrm{K}$ de l'aluminium. Ils sont comparés à des spectres théoriques obtenus par une simulation HETL. Des spectres d'absorption X (XANES et EXAFS) d'un échantillon d'aluminium froid ont été mesurés.
\end{abstract}

\section{INTRODUCTION}

Le développement de sources $\mathrm{X}$ ultra-brèves s'est remarquablement développé ces dix dernières années, motivé par l'étude dynamique de systèmes transitoires. Parmi toutes les techniques utilisant un rayonnement X, la spectroscopie d'absorption fine (XAFS, X-ray Absorption Fine Spectroscopy) offre de nombreux avantages tels que la sélectivité en éléments ou encore l'accès à la structure locale de différents états de la matière (molécules, cristaux, liquides...) [1]. Cette méthode est donc adaptée à l'étude de la matière tiède et dense, état transitoire entre la phase solide et le plasma chaud, désordonné mais caractérisé par de fortes corrélations ions - ions.

La technique XAFS requiert un spectre d'émission $\mathrm{X}$ intense et de large bande. Aujourd'hui, de telles expériences sont principalement réalisées à l'aide de rayonnement $\mathrm{X}$ synchrotron. De par leur intense émission $\mathrm{X}$, les synchrotrons sont relativement bien adaptés à la plupart de ces études. Néanmoins, la durée d'impulsion de ce type de source est limitée à environ 50-100 ps [2]. La technique dite de "slicing" offre une durée d'impulsion sub-ps au prix d'un nombre de photons X fortement réduit $[3,4]$. Les futures machines XFEL annoncent une durée d'impulsion de quelques centaines de femtosecondes, mais avec une émission $\mathrm{X}$ de gamme énergétique réduite (rayonnement quasimonochromatique) [5]. Une autre solution consiste à produire une source $\mathrm{X}$ ultra-brève issue de plasmas chauds et denses créés par une impulsion laser ultra-courte. L'émission Bremsstralhung, présentant un spectre plat, a tout d'abord été proposée pour de telles expériences [6]. Elle est néanmoins limitée par une trop faible efficacité de conversion. Il a été suggéré d'utiliser des sources de large bande spectrale issues du rayonnement de couche $\mathrm{M}$ d'éléments de $\mathrm{Z}$ élevé [7]. La durée de ces sources $\mathrm{X}$ est inférieure à la dizaine de picosecondes [8].

Nous avons cherché à interpréter et à optimiser de telles sources $\mathrm{X}$ en vue de réaliser des expériences de spectroscopie d'absorption autour du flanc K de l'Al, dans la gamme d'énergie 1,50-1,75 keV. Pour cela, nous avons étudié l'émission de couche $\mathrm{M}$ de différentes cibles ( $\mathrm{Sm}, \mathrm{Gd}$, Dy, Er et $\mathrm{Yb}$ ) en fonction des paramètres lasers (énergie, durée d'impulsion...). Des premiers spectres d'absorption $\mathrm{X}$ ont été réalisés sur des échantillons d'aluminium froid $\left(20^{\circ} \mathrm{C}\right)$ [9].

\section{DISPOSITIF EXPÉRIMENTAL}

Une série de mesures spectrales et temporelles de l'émission X a été effectuée au laboratoire CELIA, avec le laser Ti:Sapphire Aurore (cf. Fig. 1). Celui-ci délivre des impulsions au kHz, d'énergie $5 \mathrm{~mJ}$ et 


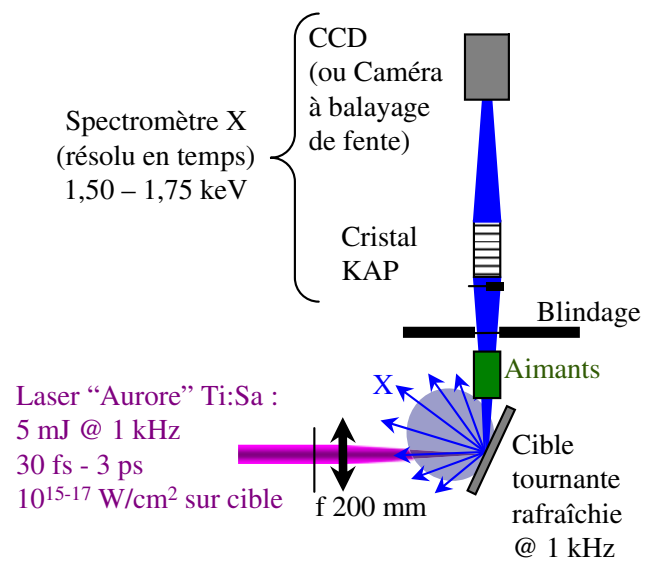

Figure 1. Dispositif expérimental.

de durée variable de $30 \mathrm{fs}$ à $3 \mathrm{ps}$ à mi-hauteur (FWHM). Une lentille f/4 focalise le laser pour obtenir une intensité de $10^{15-17} \mathrm{~W} / \mathrm{cm}^{2}$ sur cible. Les cibles sont rafraîchies à la cadence du laser pour assurer une interaction de chaque impulsion laser avec une surface propre. Les cibles utilisées n'étant pas polies, nous n'avons pas observé de différence entre les polarisations $p$ et $s$. Toutes les mesures présentées ont été obtenues en polarisation $p$, avec un angle d'incidence laser de $25^{\circ}$.

Nos spectres ont été mesurés avec deux types de spectromètre : un spectromètre intégré en temps, composé d'un cristal KAP (Potassium Acide Phtalate) et d'une caméra $\mathrm{CCD}$, placés à $90^{\circ}$ de l'axe d'incidence du laser et un spectromètre résolu en temps, pour lequel la caméra CCD est remplacée par une caméra à balayage de fente [10]. Ces 2 spectromètres sont réglés de manière à viser la gamme spectrale $1,50-1,75 \mathrm{keV}$, autour du seuil $\mathrm{K}$ de l'aluminium. Un blindage en plomb et des aimants, placés entre la cible et les spectromètres, permettent de se prémunir au mieux du bruit de fond occasionné par des $\mathrm{X}$ durs ou des électrons énergétiques. Pour protéger nos optiques des débris issus de la cible, nous avons placé des films de polypropylène devant les cristaux ainsi qu'une lame de microscope devant la lentille.

\section{DÉVELOPPEMENT D'UNE SOURCE X INTENSE ET DE LARGE BANDE SPECTRALE}

Nous avons cherché à développer et à optimiser une source $\mathrm{X}$ adaptée à la spectroscopie d'absorption autour du seuil $\mathrm{K}$ de l'aluminium $(1,559 \mathrm{keV})$. Pour cela, le spectre de notre source $\mathrm{X}$ doit présenter une émissivité élevée et une émission large bande sur environ $200 \mathrm{eV}$. Sur la figure 2 sont présentés les spectres d'émisson des éléments de Z élevé suivants : samarium ( $\mathrm{Sm}$ ), gadolinium $(\mathrm{Gd})$, dysprosium (Dy), erbium (Er) et ytterbium ( $\mathrm{Yb})$. Toutes les mesures ont été enregistrées sur la même gamme spectrale. Pour des raisons de clarté de présentation, nous avons choisi de normaliser l'axe des énergies de chaque spectre à l'énergie de liaison du flanc $M_{1} 3 s$ correspondant à l'élément étudié. Cette représentation révèle le comportement global de l'émission de couche $\mathrm{M}$ des éléments de $\mathrm{Z}$ élevé. De tels spectres, sur des gammes d'énergie plus grandes, ont déjà été observés et interprétés pour l'or $(\mathrm{Au})$, le tantale (Ta) et le gadolinium (Gd) $[11,12]$. Des structures spectrales sont identifiées aux faisceaux de transitions $4 f-3 d, 3 d-3 p$ et $5 f-3 d$ (cf. Fig. 2). La structure $4 f-3 d$, ici émise par l'erbium dans la gamme 1,50-1,75 keV, est proéminente et quasi-continue spectralement. L'erbium semble donc être le meilleur candidat pour des expériences de spectroscopie d'absorption autour du seuil $\mathrm{K}$ de l'aluminium. De manière plus générale, la figure 2 permet de se faire une idée de l'élément le plus adéquat pour des expériences de spectroscopie d'absorption dans une gamme spectrale donnée. Il suffit pour cela de 


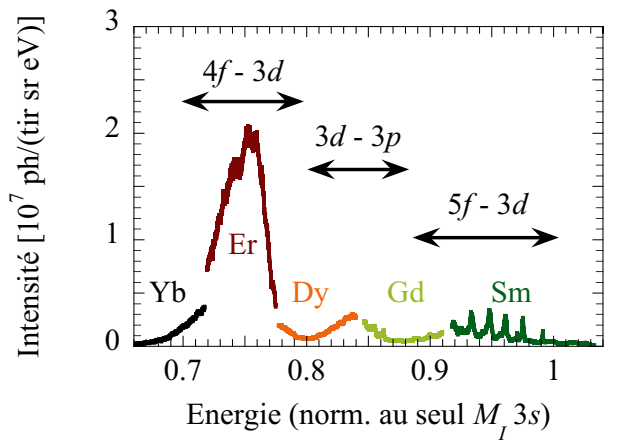

Figure 2. Spectres de rayonnement $\mathrm{X}$ de cibles de $\mathrm{Z}$ élevé (Yb, Er, Dy, Gd, Sm). L'axe des abscisses correspond à l'énergie des photons $\mathrm{X}$ normalisée à l'énergie de liaison respective du seuil $M_{1} 3 s$. Les paramètres laser sont une énergie de 5,1 mJ et une durée d'impulsion de 2,7 ps FWHM.

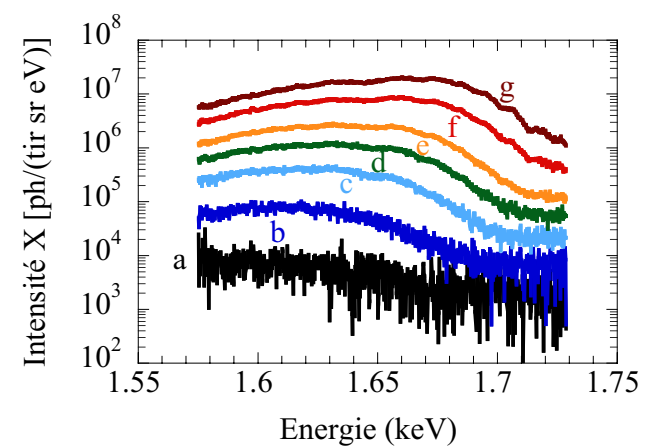

Figure 3. Spectres de rayonnement $X$ d'une cible d'erbium, pour une durée d'impulsion laser de 2,7 ps FWHM et pour différentes énergies laser : $0,6 \pm 0,1 \mathrm{~mJ}$ (a), 1,0 $\pm 0,1 \mathrm{~mJ}$ (b), 1,6 $\pm 0,1 \mathrm{~mJ}$ (c), 2,0 $\pm 0,1 \mathrm{~mJ}$ (d), $2,4 \pm 0,1 \mathrm{~mJ}(\mathrm{e}), 3,4 \pm 0,1 \mathrm{~mJ}(\mathrm{f}), 5,1 \pm 0,1 \mathrm{~mJ}(\mathrm{~g})$.

chercher l'élément dont l'énergie de liaison $M_{1} 3 s$ est suffisante pour projeter cette figure normalisée dans la gamme spectrale souhaitée.

Sur la figure 3, le spectre d'émission de l'erbium est mesuré pour des énergies laser allant de $0,6 \mathrm{~mJ}$ à $5,1 \mathrm{~mJ}$. Lorsque l'énergie laser augmente, on note un décalage spectral vers les plus hautes énergies d'environ $50 \mathrm{eV}$. Celui-ci est accompagné d'une augmentation de l'émissivité $\mathrm{X}$ de plus de 3 ordres de grandeur. Sur la figure 4, le spectre d'émission de l'erbium est cette fois-ci obtenu pour différentes durées d'impulsion laser et pour une énergie fixée à $5,1 \mathrm{~mJ}$. Le comportement observé est le même que précédemment : un décalage d'environ $100 \mathrm{eV}$ vers les plus hautes énergies ainsi qu'un accroissement de l'intensité $\mathrm{X}$ de 2 ordres de grandeur. Ce phénomène est interprété comme l'effet d'une élévation de la température dans la partie thermique du plasma [13].

Dans les conditions optimales, i.e. une énergie laser maximale $(5,1 \mathrm{~mJ})$ et une durée d'impulsion de 2,7 ps (FWHM), l'efficacité de conversion de l'énergie laser en rayonnement X est de $1 \%$. Cette valeur est déduite en intégrant angulairement l'émission X sur $2 \pi$ sr et spectralement de 1,52 à $1,72 \mathrm{keV}$.

Afin de réaliser des expériences pompe (impulsion de chauffage) - sonde (impulsion X), résolues en temps, de la transition solide - matière dense et tiède, les durées requises pour l'impulsion $\mathrm{X}$ doivent être inférieures à une dizaine de ps (temps caractéristique estimé de l'expansion hydrodynamique d'un plasma à la densité solide et chauffé à quelques $\mathrm{eV}$ ). Pour mesurer la durée d'émission de notre source $\mathrm{X}$, nous avons utilisé une caméra à balayage de fente placée derrière un cristal tronconique KAP. La résolution temporelle dépend essentiellement de la gigue temporelle entre le laser et le détecteur. 


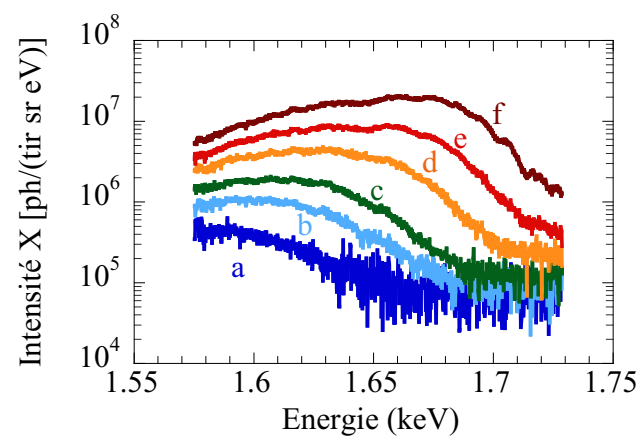

Figure 4. Spectres de rayonnement $X$ d'une cible d'erbium pour une énergie de $5,1 \mathrm{~mJ}$ et pour différentes durées d'impulsion laser : $90 \pm 20 \mathrm{fs}$ (a), $250 \pm 30 \mathrm{fs}$ (b), $450 \pm 50 \mathrm{fs}$ (c), $900 \pm 100 \mathrm{fs}$ (d), $1800 \pm 200 \mathrm{fs}$ (e), $2700 \pm 300 \mathrm{fs}(\mathrm{f})$.

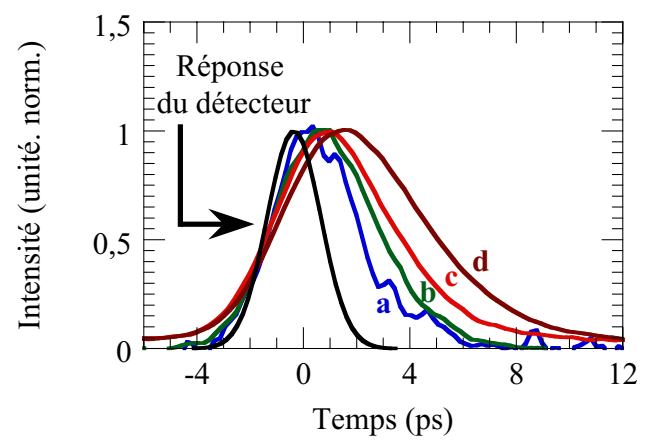

Figure 5. Mesure de la durée de l'émission $X$ d'une cible d'erbium, pour une énergie laser de 5,1 mJ et pour différentes durées d'impulsion laser : $45 \pm 10 \mathrm{fs}$ (a), $450 \pm 50 \mathrm{fs}$ (b), $900 \pm 100 \mathrm{fs}$ (c) et $2700 \pm 300 \mathrm{fs}$ (d).

Tableau 1. Durée de l'émission $X\left(\tau_{X}\right)$ en fonction de la durée d'impulsion laser $\left(\tau_{\text {las }}\right)$.

\begin{tabular}{ccc}
\hline Label (Fig. 5) & $\tau_{\text {las }}$ (fs FWHM) & $\tau_{X}$ (ps rms) \\
\hline a & $45 \pm 15$ & $1,7 \pm 0,8$ \\
b & $450 \pm 50$ & $2,3 \pm 0,4$ \\
c & $900 \pm 100$ & $2,8 \pm 0,4$ \\
d & $2700 \pm 300$ & $3,7 \pm 0,4$ \\
\hline
\end{tabular}

Elle a été mesurée pendant l'expérience à 2, $0 \pm 0,3$ ps rms. Les mesures temporelles sont présentées pour différentes durées d'impulsion laser, aprés intégration spectrale des images obtenues (cf. Fig. 5). Les durées de la source X sont rassemblées dans le tableau 1. Pour le cas optimal déterminé plus haut, i.e. une durée d'impulsion de 2,7 ps FWHM et une énergie maximale sur cible $(5,1 \mathrm{~mJ})$, la durée de l'émission X est de 3,7 ps rms. Elle est identique sur toute la gamme spectrale étudiée (1,50-1,75 keV).

Cette source $\mathrm{X}$, de large bande spectrale, intense et brève, semble donc bien adaptée à des expériences de spectroscopie d'absorption résolue en temps.

\section{SPECTRES XANES DU SEUIL K DE L'ALUMINIUM}

Nos premières expériences de spectroscopie d'absorption $\mathrm{X}$ ont été réalisées en plaçant un échantillon d'aluminium froid devant le cristal KAP du spectromètre [9]. Le spectre transmis et le spectre incident (référence sans échantillon d'aluminium) sont enregistrés simultanément à l'aide d'un seul cristal devant lequel est placé un échantillon d'aluminium sur la moité de l'angle de collection. En plaçant le détecteur 


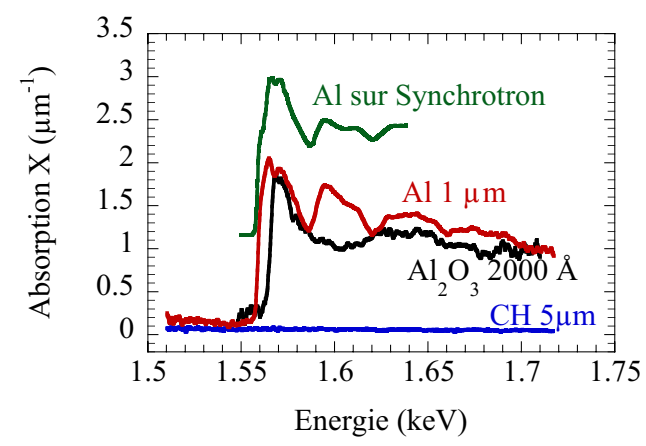

Figure 6. Spectre d'absorption X (XANES et EXAFS) de l'Al froid d'épaisseur $1 \mu \mathrm{m}\left(20^{\circ} \mathrm{C}\right)$, de $2000 \AA \AA^{\prime} \mathrm{Al}_{2} \mathrm{O}_{3}$ froid et de $5 \mu \mathrm{m}$ de $\mathrm{CH}$ et spectre XANES de l'Al froid réalisé sur une installation synchrotron.

derrière le plan focal, on obtient une résolution spatiale permettant de discriminer les spectres incident et transmis à partir desquels on déduit le spectre d'absorption X.

Des spectres d'absorption $\mathrm{X}$ ainsi mesurés sont reportés sur la figure 6. Un spectre couvrant les domaines XANES et EXAFS a été mesuré sur un échantillon d'aluminium de $1 \mu \mathrm{m}$ d'épaisseur et pour une durée d'acquisition de $30 \mathrm{~s}$ (soit 30000 tirs laser). Il se compare très bien avec une mesure XANES obtenue sur synchrotron [14]. Un spectre d'absorption de $2000 \AA \mathrm{A}^{\prime} \mathrm{Al}_{2} \mathrm{O}_{3}$ est aussi présenté. La différence avec le spectre d'absorption de l'aluminium pur est clairement identifiable : le seuil K est décalé de $6 \pm 1 \mathrm{eV}$, valeur expliquée par la proximité d'atomes d'oxygène. Grâce à un échantillon de $5 \mu \mathrm{m}$ de $\mathrm{CH}$, ne présentant aucun flanc d'absorption dans la gamme étudiée, nous mesurons des fluctuations le long du spectre d'absorption $\mathrm{X}$ de 0,3 à $0,6 \% \mathrm{rms}$, comparées à l'amplitude du flanc $\mathrm{K}$ d'absorption de l'aluminium. Ce bruit est limité essentiellement par le bruit statistique dû au nombre de photons détectés par la caméra CCD. Pour l'améliorer, il suffit d'accumuler nos mesures sur un plus grand nombre de tirs laser.

\section{CONCLUSION}

Nous avons enregistré les spectres d'émission de couche $\mathrm{M}$ d'éléments de $\mathrm{Z}$ élevé tels que le samarium, le gadolinium, le dysprosium, l'erbium et l'ytterbium. Différentes structures spectrales ont été identifiées, parmi lesquelles le faisceau de transition $4 f-3 d$, le plus émissif et étendu spectralement sur plus de $200 \mathrm{eV}$. L'erbium est l'élément qui offre la gamme spectrale du faisceau de transition $4 f-3 d$ la plus adaptée pour la spectroscopie d'absorption du seuil $\mathrm{K}$ de l'aluminium. Parallèlement, une étude de l'émission X en fonction de l'énergie laser et de la durée d'impulsion nous a permis d'optimiser la source $X$ pour une énergie laser maximale et une durée d'impulsion d'environ 3 ps. La durée de la source $X$ a alors été mesurée à 3,7 ps rms. Des premiers spectres d'absorption X d'un échantillon d'aluminium froid ont été mesurés pour des temps d'accumulation de $30 \mathrm{~s}$. Ils sont de qualité équivalente aux spectres XANES obtenus avec un rayonnement synchrotron et présentent des fluctuations de l'absorption $\mathrm{X}$ de seulement 0,3 à $0,6 \%$ par rapport à l'amplitude du flanc $\mathrm{K}$ de l'aluminium.

L'utilisation de telles sources $\mathrm{X}$ est limitée intrinsèquement à environ $5 \mathrm{keV}$, gamme spectrale d'émission de la couche $\mathrm{M}$ de l'uranium, élément de plus haut $\mathrm{Z}$ accessible naturellement. Les applications possibles restent cependant très étendues et concernent une grande variété de phénomènes ultra-rapides. Il est même envisageable de diminuer la durée de l'émission X, actuellement de quelques ps, pour atteindre des durées sub-ps, en utilisant des sources $\mathrm{X}$ issues de plasmas d'agrégats de $\mathrm{Z}$ élevés [15]. 
On se propose maintenant d'étudier l'aluminium dense et tiède. Différentes options sont alors envisageables pour atteindre cet état : par chauffage laser isochore, par chauffage par protons ou encore par choc. Toutes ces méthodes donnent accès à des états de la matière plus ou moins chauds et denses et permettent ainsi d'aborder différentes dynamiques jusqu'à la matière dense et tiède (état Warm Dense Matter, WDM). Parallèlement, des spectres XANES théoriques de l'aluminium dense et tiède [16] nous permettent d'attendre des modifications nettes des structures XANES lors de la transition solide matière dense et tiède.

\section{Remerciements}

Les auteurs remercient D. Descamps, S. Petit et C. Medina pour leur précieuse aide sur l'installation laser, ainsi que R. Bouillaud et A. Le Goff pour leur aide technique. Ce travail est soutenu par le Fond Européen de Développement Économique Régional et le Conseil Régional d’Aquitaine.

\section{Références}

[1] C. Bressler, M. Chergui, Chem. Rev. 104, 1781 (2004)

[2] M. Saes, F. van Mourik, W. Gawelda, M. Kaiser, M. Chergui, C. Bressler, D. Grolimund, R. Abela, Rev. Sci. Instrum. 75, 24 (2004)

[3] R.W. Schoenlein, S. Chattopadhyay, H.H.W. Chong, T.E. Glover, P.A. Heimann, C.V. Shank, A.A. Zholents, M.S. Zolotorev, Science 287, 2237 (2000)

[4] A. Cavalleri, M. Rini, H.H.W. Chong, S. Fourmaux, T.E. Glover, P.A. Heimann, J.C. Kieffer, R.W. Schoenlein, Phys. Rev. Lett 95, 0637405 (2005)

[5] K.J. Kim, Y. Shvyd'ko, S. Reiche, Phys. Rev. Lett. 100, 244802 (2008)

[6] T. Lee, F. Benesch, Y. Jiang, C.G. Rose-Petruck, Chem. Phys. 299, 233 (2004)

[7] P. Forget, F. Dorchies, J.C. Kieffer, O. Peyrusse, Chem. Phys. 299, 259 (2004)

[8] P. Audebert, V. Nagels, J.P. Geindre, F. Dorchies, O. Peyrusse, S. Gary, F. Girard, R. Sheperd, J.C. Gauthier, C. Chenais-Popovics, J. Quant. Spectrosc. Radiat. Transf. 81, 19 (2003)

[9] F. Dorchies, M. Harmand, D. Descamps, C. Fourment, S. Hulin, S. Petit, J. Santos, Appl. Phys. Lett. 93, 121113 (2008)

[10] C. Bonté, M. Harmand, F. Dorchies, S. Magnan, V. Pitre, J.C. Kieffer, P. Audebert, J.P. Geindre, Rev. Sci. Instrum. 78, 043503 (2007)

[11] P.G. Burkhalter, D.J. Nagel, R.R. Whitlock, Phys. Rev. A 9, 2331 (1974)

[12] J.C. Gauthier, P. Monier, P. Audebert, C. Chenais-Popovics, J.P. Geindre, Laser and Part. Beams 4, 421 (1986)

[13] M. Harmand, F. Dorchies, O. Peyrusse, D. Descamps, C. Fourment, S. Hulin, S. Petit, J. Santos, submitted to Phys. Plasmas (2009)

[14] J. Wong, G. George, I. Pickering, Z. Rek, R. M., T. Tanaka, G. Via, B. DeVries, D. Vaughan, G. Brown, Solid State Commun. 95, 559 (1994)

[15] F. Dorchies, F. Blasco, C. Bonté, T. Caillaud, C. Fourment, O. Peyrusse, Phys. Rev. Lett. 100, $205002(2008)$

[16] O. Peyrusse, J. Phys.: Condens. Matter 20, 195211 (2008) 